\title{
ЭФФЕКТИВНАЯ ДИСПАНСЕРИЗАЦИЯ И АДЕКВАТНАЯ ФИЗИЧЕСКАЯ АКТИВНОСТЬ КАК СОСТАВЛЯЮЩИЕ АНТИ-ЭЙДЖИНГОВОЙ ПРОГРАММЫ НА ПРЕДПРИЯТИИ БЕЛГОРОДСКОЙ ОБЛАСТИ
}

\section{EFFECTIVE PROPHYLACTIC MEDICAL EXAMINATION AND ADEQUATE PHYSICAL ACTIVITY AS COMPONENTS OF THE ANTI-AGING PROGRAM AT THE ENTERPRISE OF THE BELGOROD REGION}

Ищенко Карина Александровна Горелик С ветлана Гиршевна Милютина Елена Валерьевна Барабанщиков Александр Александрович

щБелгородский государственный национальный исследовательский университет
Ishchenko Karina Alexandrovna

Gorelik Svetlana Girshevna

Miliutina Elena Valerievna

Barabanshchikov Alexandr Alexandrovich

щBelgorod State National Research University

E-mail: miromax93@ mail.ru

\section{Резюме}

В статье представлены результаты исследования по оценке эффективности диспансеризации, проводимой на предприятии Белтородской области, и результаты анкетирования сотрудников этото предприятия на вопрос о наличии у них хронических неинфекционных заболеваний и частоте занятий физической культурой. Полученные данные будут использованы для разработки и внедрения анти-эйджинтовой протраммы на предприятие Белтородской области, которая в свою очередь будет являться одной из мер профилактики старения. По результатам исследования можно сделать вывод о низкой эффективности проводимой диспансеризации среди сотрудников предприятия Белтородской области. Так же следует отметить, что большинство респондентов отмечает у себя наличие хронических неинфекционных заболеваний. Что касается частоты занятий физической культурой, то основная составляющая респондентов выполняют физические упражнения в среднем один раз в неделю, но доля сотрудников, ответивших, что не занимаются совсем, достаточно велика. В дальнейшем нами будет разработана корпоративная анти-эйджинтовая протрамма для предприятия Белтородской области.

Ключевые слова: диспансеризация, хронические неинфекционные заболевания, физическая активность, анти-эйджинтовые протраммы, профилактика старения

\section{Summary}

The article presents the results of a study to assess the effectiveness of clinical examination carried out at an enterprise in the Belgorod region, and the results of a survey of employees of this enterprise on the question of whether they have chronic noninfectious diseases and the frequency of physical education. The obtained data will be used for the development and implementation of an anti-aging program at the enterprise of the Belgorod region, which in turn will be one of the measures to prevent aging. According to the results of the study, it can be concluded that the effectiveness of the ongoing medical examination among employees of the enterprise in the Belgorod region can be concluded.

It should also be noted that the majority of respondents note that they have chronic non-communicable diseases. As for the frequency of physical education, the main component of the respondents do physical exercises on average once a week, but the share of employees who answered that they do not do it at all is quite large. In the future, we will develop a corporate anti-aging program for an enterprise in the Belgorod region.

Key words: clinical examination, chronic non-communicable diseases, physical activity, anti-aging programs, prevention of aging 
Ищенко К.А., Горелик С.Г., Милютина Е.В., Барабанщиков А.А. Эффективная диспансеризация и адекватная физическая активность как составляющие анти-эйджинговой программы на предприятии Белгородской области // Innova. DOI: $10.21626 /$ innova/2020.4/03 2020.-№ 4(21).-C.12-17.

\section{Введение}

Одной из важнейших проблем, которая остро стоит перед системой здравоохранения РФ и здравоохранением всего мира, является проблема глобального старения населения. Наблюдается тенденция увеличения продолжительности жизни. В РФ средняя продолжительность жизни по результатам первого полугодия 2019 года увеличилась на 0,8 года и составляет 73,7 года. В частности, у женщин средняя продолжительность жизни 78,5 года, а у мужчин - 68,6 лет.

Данный феномен послужил одним из факторов для бурного развития геронтологии и гериатрии. О днако, данное направление науки до настоящего времени было сконцентрировано на оказании помощи населению в возраст 60 лет и старше. Но, клинико-эпидемиологические данные указывают, что не менее $40 \%$ населения зрелого возраста имеют признаки преждевременного старения. О важности этого свидетельствует Первый доклад ВОЗ по старению (2014), в котором люди зрелого возраста выделяются как отдельная целевая группа профилактического гериатрического воздействия [1].

Важным инструментом диагностики хронических неинфекционных заболевания, факторов риска их возникновения является диспансеризация, Диспансеризация - это комплекс мер, включающий в себя профилактический медицинский осмотр и дополнительные методы обследований, направленные на выявление хронических неинфекционных заболеваний, а также риска их развития [2]. В Российской Федерации диспансеризация выполняется по приказу от 13 марта 2019 года № 124н «Об утверждении порядка проведения профилактического медицинского осмотра и диспансеризации определенных групп взрослого населения» [2]. Особенно важна диспансеризация, ежегодно проводимая администрацией предприятия в силу охвата всего возрастного диапазона, а не определенных групп населения.

$$
\text { Хронические }
$$
неинфекционные заболевания тесно связаны с преждевременным старением. Считается, что старение способствует возникновению заболеваний, хотя, новые результаты исследований показывают, что обратное также может быть верным: болезни, а также их лечение могут приводить $\mathrm{k}$ преждевременному старению [3].

Поэтому раннее выявление хронических неинфекционных заболеваний при помощи
Ishchenko K. A., Gorelik S. G., Mlllutina E. V., Barabanshchikov A. A. Effective prophylactic medical examination and adequate physical activity as components of the antl-aging program at the enterprise of the Belgorod region // Innova. - 2020. - No. 4 (21). P. 12-17.

диспансеризации является одной из мер профилактики преждевременного старения населения.

Так же важным составляющим звеном здоровья является адекватная физическая активность. Известно, что регулярные физические упражнения являются профилактикой многих заболеваний и являются важным составляющим здорового образа жизни наряду с правильным питанием. Но регулярная физическая активность является еще и профилактикой преждевременного старения. Естественной чертой старения можно считать такие явления как остеопороз и саркопения. Однако, улучшение образа жизни, в том числе выполнение физических упражнений, позволяет эффективно бороться с данными патологическими состояниями [4]. Имеются данные, что физические упражнения полезны для здоровья человека во все жизненные периоды, только если они имеют умеренную интенсивность. В таком случае физические упражнения оказывают системное и комплексное действие на здоровье человека, в том числе на регуляцию окислительно-восстановительного гомеостаза и воспаление [5]. Таким образом, адекватная и умеренная физическая активность так же является составляющей профилактики старения.

На данный момент в РФ нет единого комплекса мероприятий по улучшению здоровья корпоративного характера. Руководствуясь зарубежным опытом, можно предположить, что корпоративные анти-эйджинговые программы смогут занять эту нишу. Например, в Германии внедренные программы по укреплению здоровья на рабочем месте принесли определенные выгоды, такие как сокращение числа прогулов по болезни и повышение производительности [6]. Хотелось бы дать определение данному понятию. Итак, корпоративная анти-эйджинговая программа - это организованная и оплачиваемая работодателем программа, направленная на поддержание сотрудников в деятельности по улучшению состояния здоровья, снижению рисков здоровья, ведению здорового образа жизни и повышению их индивидуальной эффективности на работе [7].

С ледовательно, полученные нами в ходе исследования данные послужат опорой для разработки, а затем и внедрения корпоративных анти-эйджинговых программ на предприятия Белгородской области.

Цель: оценить эффективность проводимой на предприятии Белгородской 
области диспансеризации и частоту занятий физической культурой среди сотрудников данного предприятия.

\section{материалы и методы}

Для достижения цели исследования была разработана авторская анкета, включающая в себя вопросы о понимании и применении практик здорового образа жизни. Так же проводился анализ медицинской документации по результатам проводимой на предприятии Белгородской области диспансеризации сотрудников данного предприятия. В ходе исследования проанализированы данные 400 сотрудников предприятия Белгородской области, исследование проводилось на базе АО «Белгородский хладокомбинат».

\section{Результаты и обсуждение}

По результатам опроса выявлена гендерная структура, которая составляет 37,10\% мужчин, 62,90\% женщин. Определена возрастная структура: 18-29 лет- 18,95\%, 30-39 лет - 31,24\%, 40-49 лет - 28,23\%, 50-59 лет - 18,55\%, от 60 лет и старше - 3,03\%. В результате анкетирования были получены данные 0 имеющихся хронических неинфекционных заболеваний у сотрудников предприятия Белгородской области. Число сотрудников с патологией сердечнососудистой системы 6,67\%, в том числе имеющие гипертоническую болезнь - 8,53\%, заболевания дыхательной системы отмечает 0,80\% опрошенных, заболевания желудочнокишечного тракта имеет 6,13\% респондентов, из которых патологию печени составляет 0,53 \%, $8,00 \%$ сотрудников отметили наличие заболеваний опорно-двигательной системы, $2,40 \%$ составляет эндокринная патология, неврологические заболевания имеют 4,00\% опрошенных, почечная и гинекологическая патологии составляют $2,13 \%$ и $5,07 \%$ соответственно, аллергические заболевания отмечают у себя 5,60\% сотрудников, хирургическая патология составила 5,33\%, отсутствие перечисленных в анкете заболеваний отметили у себя $13,07 \%$ респондентов, затруднились ответить 12\%. Более подробно сложившуюся картину отражает диаграмма на рисунке 1.

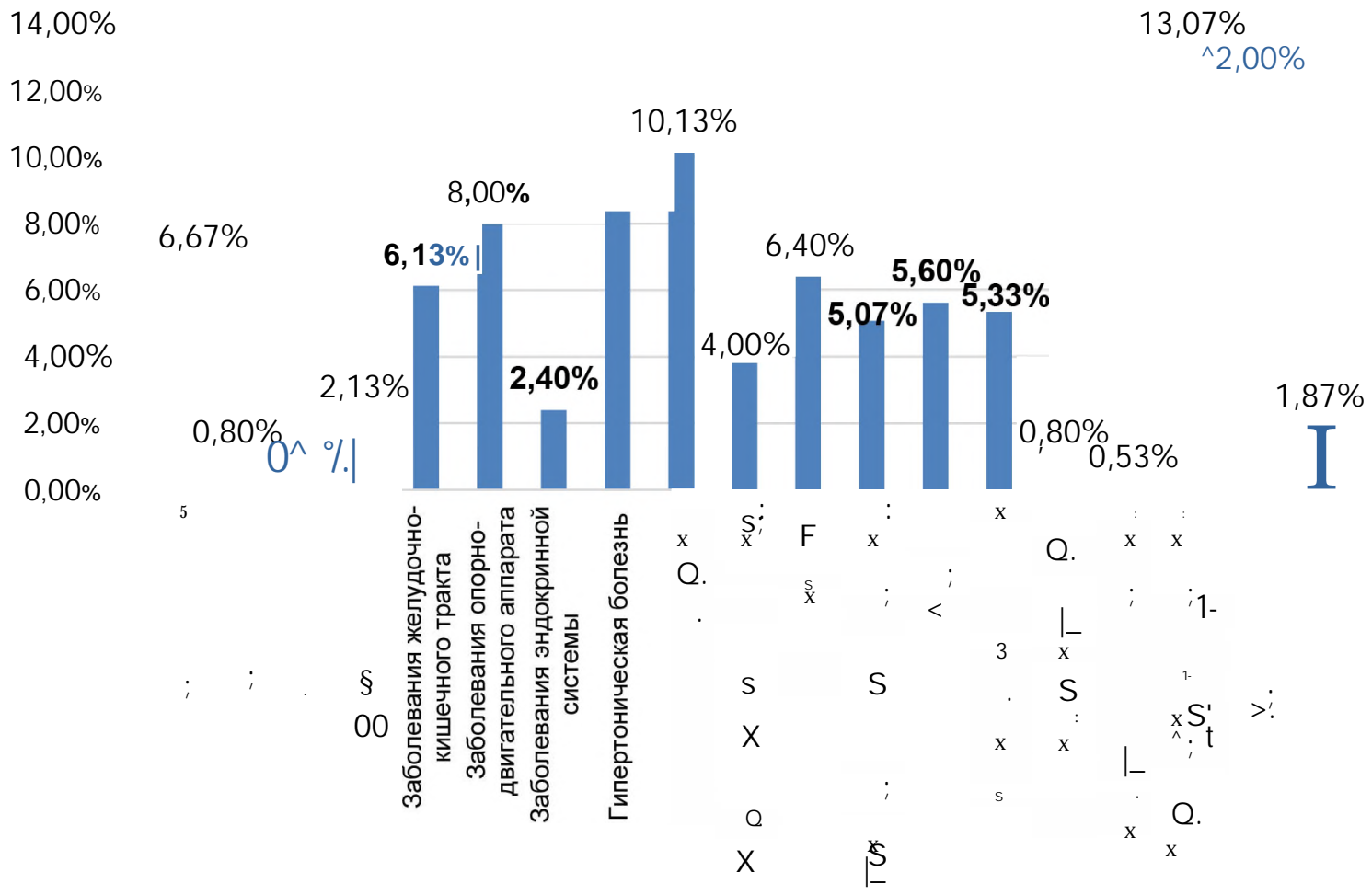

Рис. 1 Хронические заболевания сотрудников предприятия Белгородской области

Патология сердечно-сосудистой системы, включающая в себя гипертоническую болезнь занимает лидирующую позицию среди выявленных заболеваний у сотрудников предприятия Белгородской области.

В патогенезе таких патологических процессов как заболевания сердечно-сосудистой системы, активное участие принимают явления окислительного стресса и клеточного старения. Кроме того, факторы риска сердечно-сосудистых заболеваний такие как ожирение, метаболический синдром, дислипидемия связаны с воспалительным путем, опосредованным IL-1a, IL-6, IL-8, и повышенным 
клеточным старением [8]. Профилактика и раннее выявление заболеваний сердечнососудистой системы - превентивная мера преждевременного старения.

Из анализа медицинской документации, а именно результатов диспансеризации сотрудников получены следующие данные: при прохождении медицинского осмотра у 4,83\% сотрудников была установлена сердечнососудистая патология, в том числе гипертоническая болезнь - 6,55\%, заболевания дыхательной системы встречаются в 1,03\% случаев, заболевания желудочно-кишечного тракта, в той числе печени диагностированы у
3,10\% и 0,34\% соответственно, заболевания опорно-двигательного аппарата отмечены у 4,14\% сотрудников, заболевания глаз - 4,14\%, гинекологические заболевания - 5,52\%, неврологические заболевания диагностированы у 2,07\% респондентов, при прохождении диспансеризации не выявлено хронических неинфекционных заболевания у $32,41 \%$ сотрудников, $\quad 21,03 \% \quad$ проходили диспансеризацию в другом месте.

Процентное соотношение выявленных хронических неинфекционных заболеваний отражено на рисунке 2.

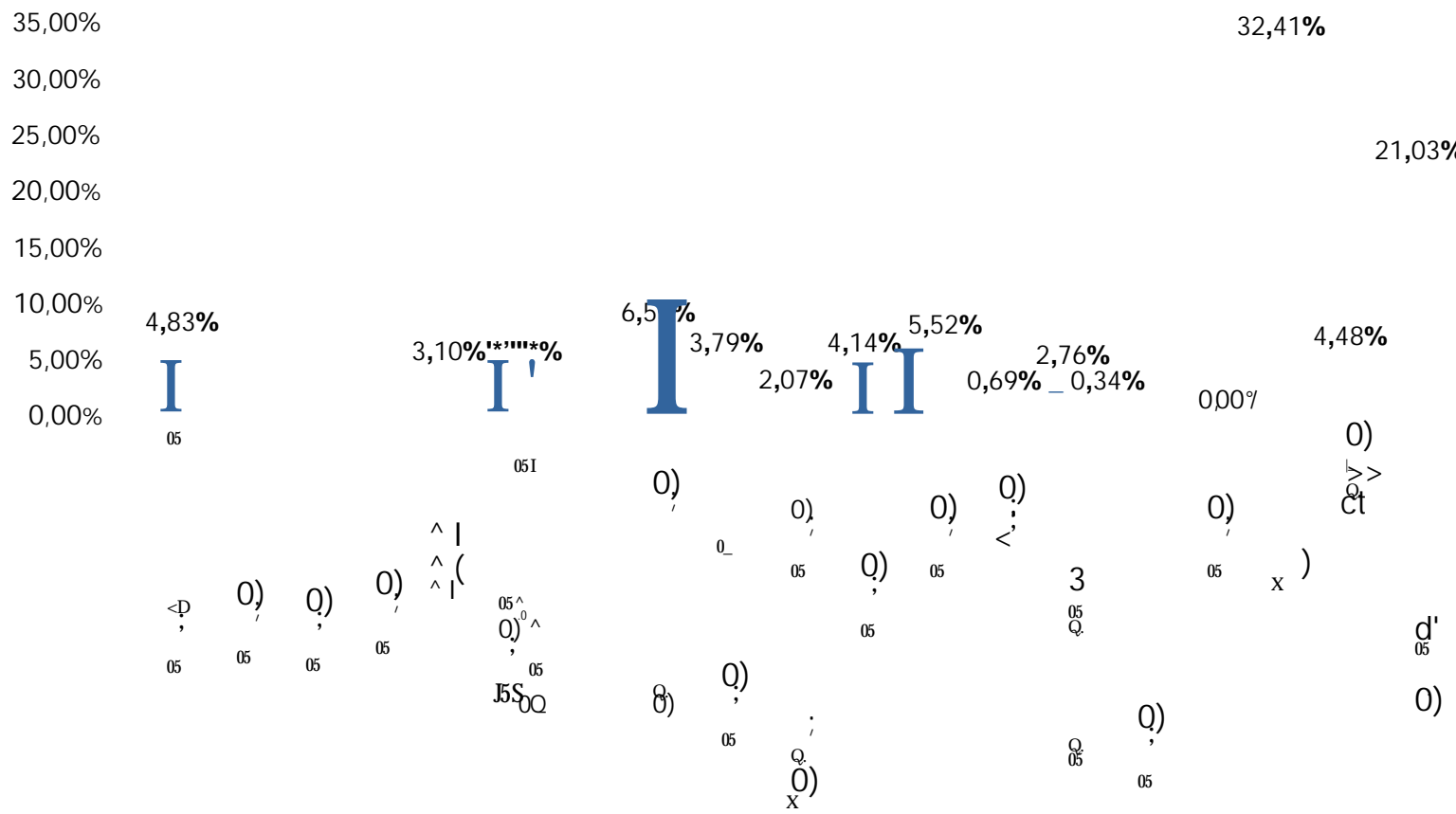

Р ис. 2 Хронические заболевания сотрудников предприятия, обнаруженные при прохождении диспансеризации

Полученные данные свидетельствуют о проведенной диспансеризации как о малоэффективном мероприятии. Хотя, как уже отмечалось ранее, диспансеризация выявляет не только хронические неинфекционные заболевания, но и факторы риска к их возникновению. Одним из таких факторов является метаболический синдром. Пациенты с данным синдромом ведут малоподвижный образ жизни, неправильно питаются. Данные факторы, самостоятельно либо в совокупности, могут привести к развитию преждевременного старения. Так же было доказано, что терапия метаболического с помощью метформина не только приводит к снижению висфатина маркера преждевременного, старения, но и дает возможность проведения мероприятий против преждевременного старения у пациентов с сахарным диабетом 2-го типа при метаболическом синдроме [9].

Как было описано выше, хронические неинфекционные заболевания

преждевременное старение образуют замкнутый круг, для разрыва которого на предприятии должна проводиться эффективная по своей мощности диспансеризация сотрудников.

Что касается вопроса о частоте занятий физической культурой среди сотрудников предприятия Белгородской области получены были следующие данные: 8,87\% занимаются ежедневно, 2-3 раза в неделю - 18,95\%, 12,50\% сотрудников выполняют физические упражнения 1 раз в неделю, 10,08\% - 1-2 раза в месяц, реже одного раза в месяц - $\quad 7,66 \%, 27,02 \%$ респондентов не занимаются физической культурой, затруднились ответить - 14,92\%.

Доля опрошенных сотрудников, регулярно занимающихся физической культурой 
хотя бы один раз в неделю, составила $40,32 \%$, что больше доли сотрудников, которые совсем не выполняют $13,30 \%$. физические упражнения, на

$27,02 \%$

области

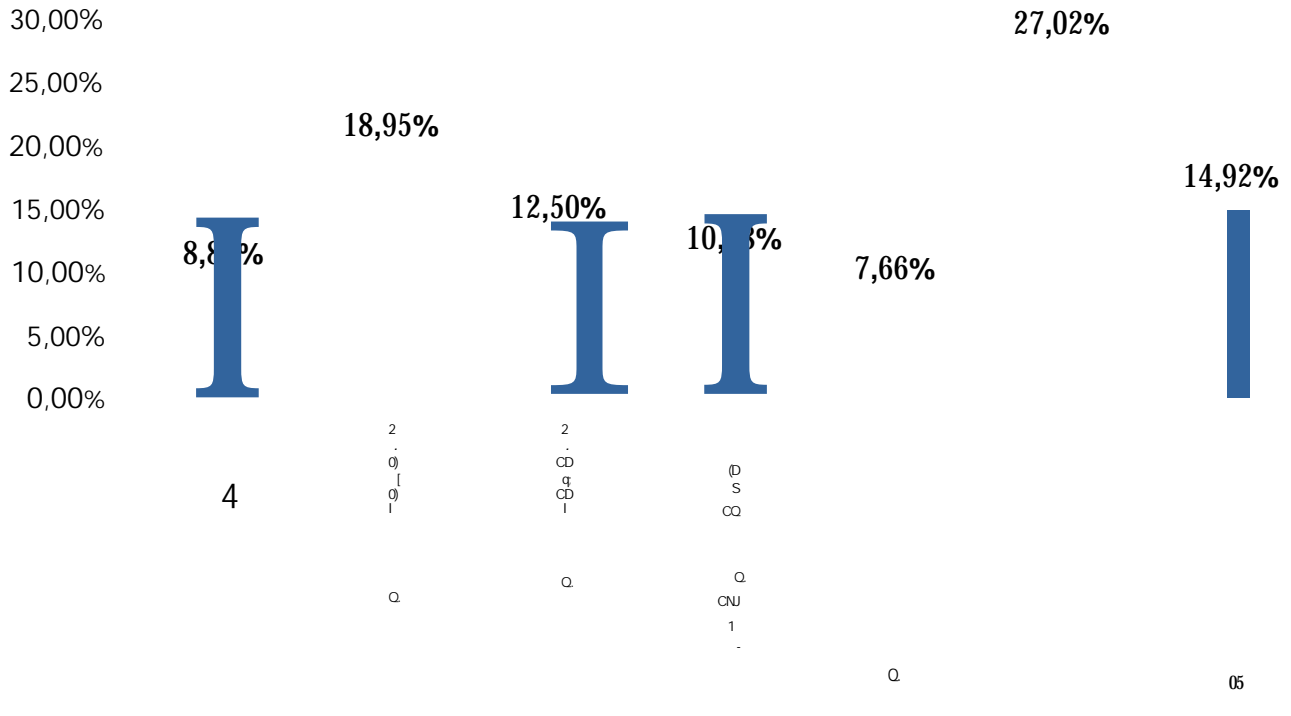

Рис. 3 Частота занятий физической культурой сотрудников предприятия Белгородской

Стоит отметить крайне важную роль физической культуры в поддержании и соблюдении практик здорового образа жизни и огромную роль в профилактике преждевременного старения. В литературе имеются данные, что умеренная физическая активность имеет ряд преимуществ для здоровья человека, а ее снижение оказалось одним из сильных и независимых факторов, связанных с ростом заболеваемости и смертностью. Среди положительных эффектов физических упражнений отмечается позитивное влияние на продолжительность жизни и замедление возникновения некоторых хронических неинфекционных заболеваний. В частности, было замечено, что умеренная физическая активность замедляет прогрессирование старения тканей и органов, например, головного мозга и сердечно-сосудистой системы путем сосудистых, структурных и нейромолекулярных изменений. Данные изменения в свою очередь замедляют развитие инсулинорезистентности, воспаления и окислительного стресса [5].

Таким образом, проанализировав полученные данные, можно сделать вывод о крайней важности мер, описанных выше. K сожалению, контроль эффективности проводимой диспансеризации и контроль частоты занятий физической культурой сотрудников предприятия Белгородской области крайне затруднителен. Поэтому нами была предложена идея разработки и внедрения такой программы, которая бы в полном объеме являлась мерой профилактики преждевременного старения и была полностью контролируема. Корпоративная антиэйджинговая программа полностью соответствует нашим требованиям. В Росиийской Федерации на данный момент подобные программы отсутствуют. Так же в современной русскоязычной литературе данные о подобных мерах не встречаются. Так, опираясь на зарубежные исследования, хотелось бы отдельно отметить корпоративный оздоровительный проект «Ferrari Formula Benessere», который доказал свою эффективность в улучшении профиля риска сердечно-сосудистых заболеваний и кардиореспираторной патологии среди физически активных сотрудников, работающих в автомобильной компании Ferrari в Маранелло, Модена (Италия) [10]. Еще один пример положительного влияния корпоративной антиэйджинговой программы, внедеренной американскими учеными, показывает, что среди сотрудников крупной складской розничной компании подобная программа на рабочем месте привела к значительно большему количеству положительных форм поведения в отношении здоровья среди лиц, участвовавших в эксперименте, по сравнению с сотрудниками, не принимавших участия [11].

Что касается составляющих антиэйджинговой программы, то учитывая опыт американских коллег, программа может состоять из оценки здоровья или риска для здоровья, предлагаемой работодателем, которая обычно представляет собой ежегодный или полугодовой медицинский осмотр, включающий определение индекса массы тела, артериального давления. 
уровня холестерина и сахара крови сотрудника. Отдельное внимание уделяется наличию вредных привычек, таких как курение и употребление алкоголя. Так же работодателем предлагаются посещение тренажерного зала, бассейна, организовавыется праильное питание сотрудников. Кроме того, сотрудники, выполняющие подобную программу получают определенные бонусы, финансового либо иного характера [12]. Что касается стран с низким или средним уровнем дохода, то корпорации в Индии и Мексике уже реализуют ряд оздоровительных программ, ориентирующихся на лечение хронических неинфекционных заболеваний сотрудников. Но программ по общему укремплению здоровья или снижению контролируемых факторов риска их возникновения также не существует [13].

\section{Выводы}

Путем

анализа

медицинской документации нами была выявлена низкая эффективность проведенной диспансеризации, по результатам анкетирования проанализированы и определены имеющиеся хронические неинфекционные заболевания и частота занятий физической культурой сотрудников предприятия Белгородской области. Нами была показана связь между имеющимися хроническими заболеваниями, уровнем достаточной физической активности и преждевременным старением.

Поэтому в конечном итоге, разработка и внедрение анти-эйджинговых программ на предприятия нашей страны крайне необходимы, потому как подобные программы будут решать важнейшую проблему по профилатике преждевременного старения, учитывая динамику глобального постарения населения.

\section{Список литературы}

1. Шабалин В.Н., 2010; Фролова Е.В., 2012; Прощаев К.И., Ильницкий А.Н., 2011, 2012, 2015; Ткачева O.H., 2015; Khavinson V. и соавт., 2013; Trofimova S., 2019.

2. Приказ № 124н от 13 марта 2019 года «Об утверждении порядка проведения профилактического медицинского осмотра и диспансеризации определенных групп взрослого населения».

3. Brian K Kennedy, Shelley L Berger, Anne Brunet, J udith Campisi , Ana Maria Cuervo, Elissa S Epel, Claudio Franceschi, Gordon J Lithgow, Richard I Morimoto, J effrey E Pessin , Thomas A Rando, Arlan Richardson, Eric E Schadt
Tony Wyss-Coray, Felipe Sierra, Geroscience: linking aging to chronic disease, 2016.

4. Review Best Pract Res Clin Obstet Gynaecol 2013 Oct;27(5):673-88. Musculoskeletal ageing and primary preventionю Anders Nedergaard, Kim Henriksen, Morten A Karsdal, Claus Christiansenю Affiliations expand.

5. Mediators Inflamm. 2018. AntiInflamm-Ageing and/or Anti-Age-Related Disease Emerging Treatments: A Historical Alchemy or Revolutionary Effective Procedures? Carmela Rita Balistrericorresponding author.

6. Crganizational-level determinants of participation in workplace health promotion programs: a cross-company study Liesa Marie Lier, Christoph Breuer, Soren Dallmeyer, 2019.

7. Колосницына, М.Г. Экономика здравоохранения [Текст] / М.Г.Колосницына, И.М.Шейман, С.В.Шишкин. - М.: изд.дом ГУ ВШЭ, 2009 г. -479 C.

8. Maria Liguori, Gennaro Russo, Francesco Curcio, Giulia Bulli, Luisa Aran, David Della-Morte, Gaetano Gargiulo, Gianluca Testa, Francesco Cacciatore, Domenico Bonaduce, Pasquale Abete, Cxidative stress, aging, and diseases, Clin Interv Aging, 2018.

9. Чернышева Е.Н., Спыт

применения комплексного лечения для коррекции процессов преждевременного

старения у пациентов с метаболическим синдромом. Современные проблемы науки и образования. Издательский дом «Академия естествознания», Пенза, с. 793, 2015 год.

10. Ferrari Corporate Wellness Program: Results of a Pilot Analysis and the "Drag" Impact in the Workplace Alessandro Biffi, Fredrick Fernando, Paolo Emilio Adami, Michele Messina, Felice Sirico, Fernando Di Paolo, Roberta Coluccia, Claudio Borghi, Flavio D'Ascenzi \& Massimo Volpe High Blood Pressure \& Cardiovascular Prevention volume 25, pages261-266(2018)Cite this article, 2018.

11. Randomized Controlled Trial J AMA 2019 Apr 16;Effect of a Workplace Wellness Program on Employee Health and Economic Cutcomes: A Randomized Clinical Trial Zirui Song, Katherine Baicker.

12. Corporate wellness programs: implementation challenges in the modern american workplace. Bahaudin G Mujtaba, Frank J Cavico. Affiliations expand, 2013.

13. Heather Wipfli , Kristin Dessie Zacharias , Nuvjote Nivvy Hundal, Luz Myriam Reynales Shigematsu, Deepika Bahl, Monika Arora , Shalini Bassi, Shubha Kumar, Workplace wellness programming in low-and middle-income countries: a qualitative study of corporate key informants in Mexico and India. Global Health, 2018. 